Louisiana State University

LSU Digital Commons

$1-10-2014$

\title{
Species coexistence and the dynamics of phenotypic evolution in adaptive radiation
}

\author{
Joseph A. Tobias \\ University of Oxford \\ Charlie K. Cornwallis \\ University of Oxford \\ Elizabeth P. Derryberry \\ Louisiana State University \\ Santiago Claramunt \\ Louisiana State University \\ Robb T. Brumfield \\ Louisiana State University
}

See next page for additional authors

Follow this and additional works at: https://digitalcommons.Isu.edu/biosci_pubs

\section{Recommended Citation}

Tobias, J., Cornwallis, C., Derryberry, E., Claramunt, S., Brumfield, R., \& Seddon, N. (2014). Species

coexistence and the dynamics of phenotypic evolution in adaptive radiation. Nature, 506 (7488), 359-363.

https://doi.org/10.1038/nature12874

This Article is brought to you for free and open access by the Department of Biological Sciences at LSU Digital Commons. It has been accepted for inclusion in Faculty Publications by an authorized administrator of LSU Digital Commons. For more information, please contact ir@lsu.edu. 


\section{Authors}

Joseph A. Tobias, Charlie K. Cornwallis, Elizabeth P. Derryberry, Santiago Claramunt, Robb T. Brumfield, and Nathalie Seddon 


\title{
Species coexistence and the dynamics of phenotypic evolution in adaptive radiation
}

\author{
Joseph A. Tobias $^{1 *}$, Charlie K. Cornwallis ${ }^{1,2 *}$, Elizabeth P. Derryberry ${ }^{3,4}$, Santiago Claramunt ${ }^{3,5,6}$, Robb T. Brumfield B $^{3,5}$ \\ \& Nathalie Seddon ${ }^{1}$
}

\begin{abstract}
Interactions between species can promote evolutionary divergence of ecological traits and social signals ${ }^{1,2}$, a process widely assumed to generate species differences in adaptive radiation ${ }^{3-5}$. However, an alternative view is that lineages typically interact when relatively old $^{6}$, by which time selection for divergence is weak $^{7,8}$ and potentially exceeded by convergent selection acting on traits mediating interspecific competition'. Few studies have tested these contrasting predictions across large radiations, or by controlling for evolutionary time. Thus the role of species interactions in driving broadscale patterns of trait divergence is unclear ${ }^{10}$. Here we use phylogenetic estimates of divergence times to show that increased trait differences among coexisting lineages of ovenbirds (Furnariidae) are explained by their greater evolutionary age in relation to non-interacting lineages, and that - when these temporal biases are accounted for-the only significant effect of coexistence is convergence in a social signal (song). Our results conflict with the conventional view that coexistence promotes trait divergence among co-occurring organisms at macroevolutionary scales, and instead provide evidence that species interactions can drive phenotypic convergence across entire radiations, a pattern generally concealed by biases in age.
\end{abstract}

Phenotypic divergence through species interaction is one of the oldest concepts in evolutionary biology. The underlying mechanism-first called 'divergence of character', now generally known as character displacement ${ }^{11}$-is compellingly simple: divergent phenotypes should be favoured when closely related species interact to minimize the costs of ecological competition, misdirected aggression or hybridization ${ }^{12}$. This deterministic mechanism of selection, acting on the ecological and social traits of individuals and operating simultaneously across species, is believed to contribute to pervasive macroevolutionary patterns, including the tree-like structure of trait divergence over entire radiations ${ }^{10,13,14}$ and the non-random morphological differences found almost universally among co-occurring lineages ${ }^{14,15}$. However, although the importance of character displacement as a microevolutionary process is well documented in pairs or small numbers of species ${ }^{1,2,16}$, the broader implications for macroevolution are uncertain.

The main problem is that different processes can result in similar broad-scale patterns of phenotypic variation. When viewed across species, the key prediction of character displacement is that trait differences will be greater in coexisting (that is, sympatric) than non-interacting (allopatric) lineages ${ }^{14}$. However, because allopatric speciation is the norm, lineages may already be ancient by the time they interact in sympatry, particularly if range overlap is constrained by competitive exclusion ${ }^{17}$. Thus, greater phenotypic divergence among sympatric species may simply reflect trait differences acquired in allopatry, and accentuated by the 'ecological sorting' of pre-existing phenotypes ${ }^{18,19}$. Moreover, this biogeographical pattern could also obscure the adaptive convergence of interacting competitors?.

These opposing hypotheses can only be tested by assessing phenotypic differences among related lineages in the context both of geographical space and of evolutionary time ${ }^{12,17}$. Such tests are required across broad samples of species to ensure that results do not reflect chance events or special cases. This is particularly critical given that most studies demonstrating character displacement have focused on young or speciespoor systems, such as lakes and archipelagos, characterized by early sympatry $^{12}$. The most immediate priority is to quantify phenotypic divergence in a spatial and temporal framework, and across numerous lineages with comparable functional traits, including both resource-exploiting traits and social signals. This has not been achieved so far because of the challenges posed by sampling across extensive radiations.

To address this issue, we examined trait divergence in relation to interactions among 350 lineages of ovenbirds, a radiation of tracheophone suboscine birds that has evolved into a remarkable variety of phenotypes over the past $35 \mathrm{Myr}$ (Extended Data Fig. 1). We estimated divergence in three key functional traits associated with competition (beaks), locomotion (tarsi) and social interaction (songs) (Fig. 1). The beak is tightly linked to resource acquisition ${ }^{15}$; tarsus length provides an independent index of foraging niche and body $\operatorname{size}^{20}$; and songs provide insight into both reproductive and agonistic interactions because they function in mate attraction and territoriality ${ }^{2,9}$. Importantly, all lineages of ovenbirds share the same basic ecological niche (insectivory), and their songs, unlike those of most passerine birds, are structurally simple and apparently innate (Extended Data Figs 2 and 3 and Supplementary Information).

Comparing divergence between each ovenbird lineage and its closest relative in sympatry $(n=270)$ and allopatry $(n=249)$ showed that sympatric lineages have undergone significantly greater divergence than allopatric lineages in beaks (linear mixed model (LMM): $F_{1,305}=49.65$; $P<0.001)$, tarsi $\left(F_{1,302}=30.95 ; P<0.001\right)$ and songs $\left(F_{1,285}=6.90\right.$; $P=0.009$ ) (Fig. 1c-e and Supplementary Table 3 ). Similar findings are widespread and often interpreted as evidence for interspecific competition or character displacement (see, for example, refs 14, 21). However, it is possible that these differences between sympatry and allopatry are simply caused by disparities in evolutionary age, as phylogenetic data (Fig. 2) indicate that closest relatives in sympatry are on average 2.3 times older than those in allopatry $\left(F_{1,290}=286.1 ; P<0.0001\right)$ (Supplementary Table 3, Fig. If and Extended Data Fig. 4). As is typical for vertebrates, ovenbird lineages therefore undergo an extended allopatric phase preceding secondary contact, a temporal pattern often proposed to reflect competitive exclusion among lineages with conserved ecological niches ${ }^{6,7,19}$.

When we controlled for this difference in age between sympatric and allopatric lineages, the association between coexistence and divergence was removed (Fig. 3). There was no significant effect of sympatry on divergence in tarsi (phylogenetic linear mixed model (PLMM): $F_{1,14}=0.01$; $P=0.93$; Supplementary Table 4$)$ or beaks $\left(F_{1,39}=0.33 ; P=0.57\right.$; Supplementary Table 5$)$, and the only pattern consistently detected was an increased similarity of songs in sympatry $\left(F_{1,33}=5.95 ; P=0.02\right.$; Supplementary Table 6 ). These results were robust to best-fit models of

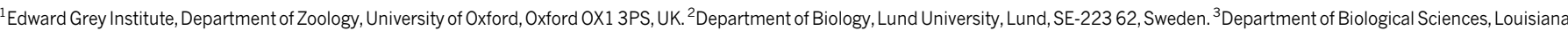

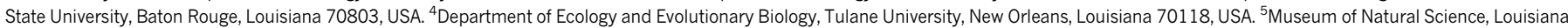
State University, Baton Rouge, Louisiana 70803, USA. ${ }^{6}$ Department of Ornithology, American Museum of Natural History, New York, New York 10024 , USA.

*These authors contributed equally to this study. 

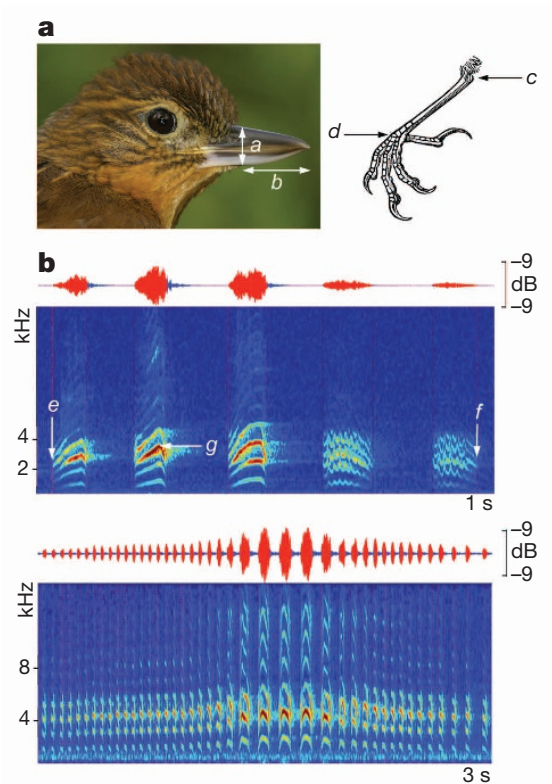

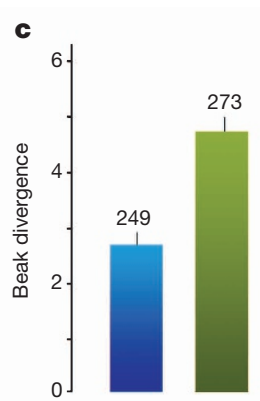

d

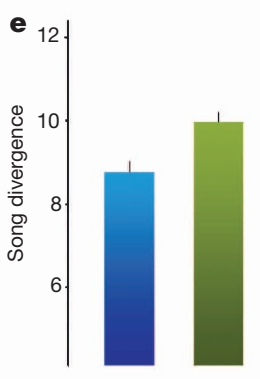

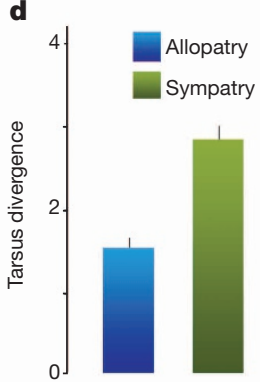

f

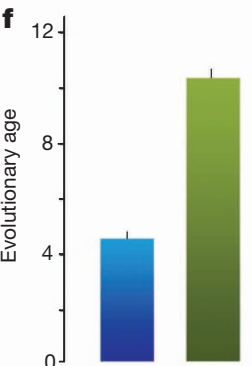

Figure $1 \mid$ Phenotypic divergence and evolutionary age in co-occurring lineages. a, Ecological traits of ovenbirds (beak depth $(a)$ and length (b); tarsus length ( $c$ to $d)$ ). $\mathbf{b}$, Social traits (spectrograms (frequency/time) and waveforms (amplitude/time) of typical songs show duration ( $e$ to $f$ ), pace ( $e$ to $f /$ number of notes) and peak frequency $(g)$ ). Closest relatives were more divergent in sympatry than allopatry in (c) beak (LMM: $P<0.001)$, (d) tarsus $(P<0.001)$, (e) song $(P=0.009)$ and $(\mathbf{f})$ evolutionary age (length of time elapsed since sharing a common ancestor $(P<0.001))$. Units of measurement: $\mathbf{c}, \mathrm{PC} 1$ scores derived from a phylogenetic principal components analysis on three beak variables; $\mathbf{d}, \mathbf{m m}$; e, distance between centroids derived from a phylogenetic principal components analysis of all acoustic traits for song; $\mathbf{f}, \mathrm{Myr}$, calculated from mitochondrial DNA sequence divergence. Bars, mean level of divergence \pm s.d.; sample sizes are given in c. (Photograph (Syndactyla striata) and spectrograms (upper, Synallaxis erythrothorax; lower, Cinclodes aricomae) by J.A.T.). trait evolution and divergence, including accounting for different models of trait evolution in sympatry versus allopatry, as well as the bounded evolution of song (Supplementary Tables 7-10). The same patterns also held when we focused on species-level taxa by removing intraspecific lineages (Supplementary Tables 11-13), and when we restricted data sets exclusively to pairs of sister species $(n=111)$ (Supplementary Table 14).
One possibility is that the classic pattern of character displacement is not detected by these analyses because it is confined to the youngest lineages, as hinted by apparent greater divergence in sympatric lineages during the initial $6 \mathrm{Myr}$ after speciation (Fig. 3a, b). To test this, we re-ran our models excluding all comparisons between lineages $>6 \mathrm{Myr}$ old. This analysis showed no divergence in sympatry for any trait, and removed significant convergence in songs (Supplementary Tables 16-18),

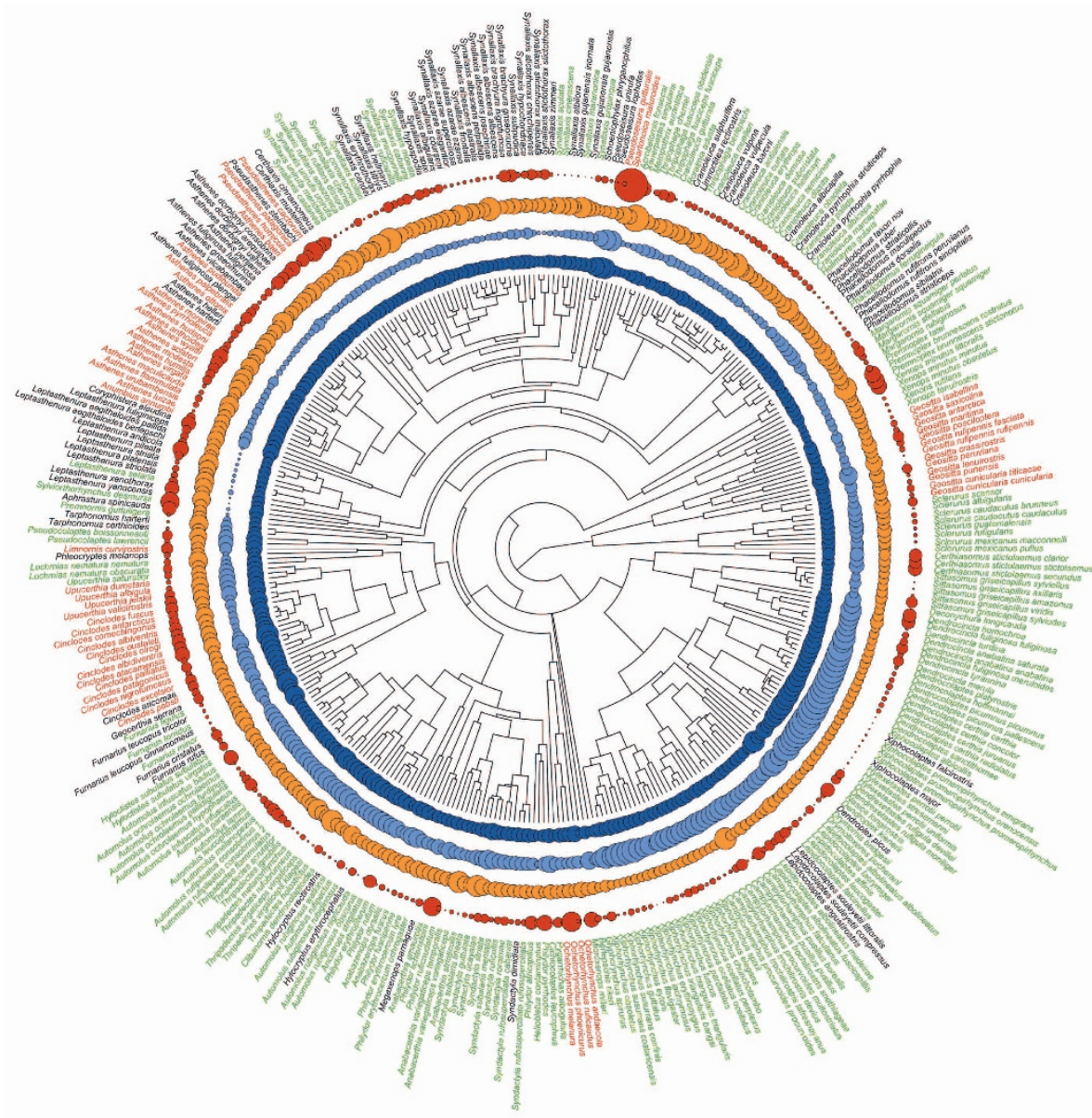

Figure $2 \mid$ Phenotype in relation to habitat and evolutionary history. The phylogram shows associations between habitat and phenotypic traits across 350 ovenbird lineages. Names are colour-coded by primary habitat structure: green, dense; black, semi-open; red, open. Dots represent variation in ecological traits (dark blue, tarsus length; light blue, beak morphology) and social traits (orange, song peak frequency; red, song pace). Dot size represents phenotypic variation (large dots, longer tarsi, larger beaks, higher peak and faster pace). 

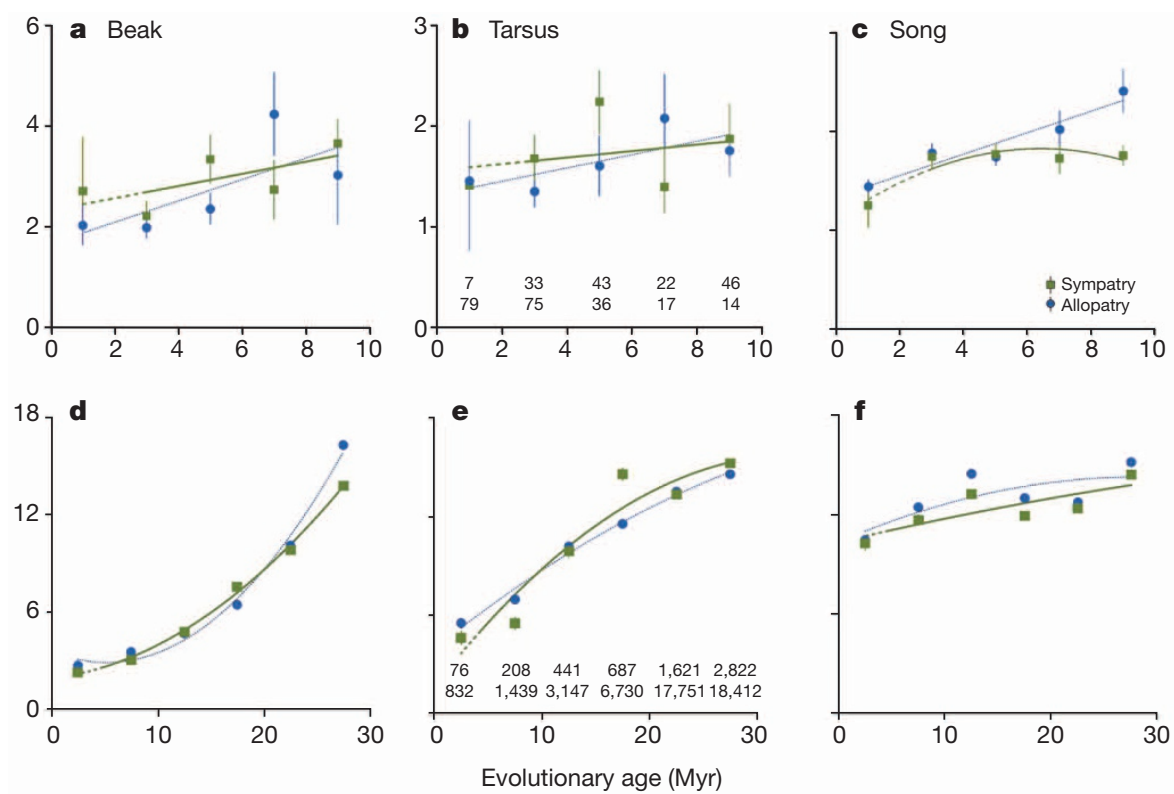

Figure 3 Comparing divergence in sympatry versus allopatry. Divergence in ovenbird beaks $(\mathbf{a}, \mathbf{d})$, tarsi $(\mathbf{b}, \mathbf{e})$ and songs $(\mathbf{c}, \mathbf{f})$ over time, calculated as Euclidean distances (units in Fig. 2) between multiple pairs of lineages. Upper panels, mean trait differences for closest relatives (sample sizes in $\mathbf{b}$ ); lower

suggesting that (1) we had not overlooked ecological or reproductive character displacement simply because they only occur in the early stages of divergence, and (2) the similarity of songs in sympatry is reduced in the youngest lineages, perhaps because the increased risk of hybridization impedes signal convergence in recently diverged species ${ }^{8}$.

To evaluate the effect of coexistence over longer timeframes, we modelled all pairwise comparisons ( $n=34,588$ pairs of lineages), again showing no significant signature of ecological character displacement when controlling for evolutionary age (Fig. 3d, e and Supplementary Tables 19 and 20). Indeed, beaks were significantly more similar in sympatry than allopatry, although this effect was weak (PLMM: $F_{1,33638}=6.04$; $P=0.01)$ and largely driven by numerous ancient sympatric species (Extended Data Fig. 5) in which beaks may be shaped by convergent adaptation to shared ecological niche ${ }^{20}$ (for example, terrestrial lineages in clades 8 (Furnariini) and 12 (Sclerurinae); see Extended Data Fig. 1). Similar environmental factors may contribute to song convergence at this broad taxonomic scale as we found positive relationships between song and habitat divergence, in line with the acoustic adaptation of songs to habitats with different transmission properties ${ }^{22}$, and between song and beak morphology, consistent with correlated evolution between ecological and social traits ${ }^{23}$ (Supplementary Table 21). Nonetheless, even after controlling for these interactions, the songs of sympatric lineages were more similar than those of allopatric lineages, an effect that was both relatively strong $\left(F_{1,33994}=27.13 ; P<0.0001\right.$; see Extended Data Fig. 4$)$ and apparently consistent regardless of the evolutionary age of interacting lineages (Fig. 3f). To verify these findings, we conducted a series of simulation tests that confirmed our results were not explained by the structure of our data or the distribution of trait divergences (Extended Data Fig. 6), or by variation in the mode of trait evolution (Extended Data Figs 7 and 8).

The preceding analyses treat sympatry and allopatry as binary variables to facilitate interpretation, in line with most previous work on character displacement. However, as the cut-off between these geographical states is somewhat arbitrary, we re-ran models including proportional range overlap as a covariate (Supplementary Tables 22-27 and Fig. 4). Focusing on closest relatives, we found no effect of range overlap on divergence in beaks (Fig. 4d) or tarsi (Fig. 4e), again rejecting the central prediction of character displacement theory. In contrast, there was a strong positive relationship between song similarity panels, data for all unique lineage combinations (sample sizes in e). Curves are fitted for significant quadratic relationships (PLMM: $P<0.05$ ). Dashed lines, small samples. Error bars, s.d. (very small in $\mathbf{d}-\mathbf{f}$ ).

and range overlap (Fig. 4f), both when considering interactions among closest relatives $\left(F_{1,304}=10.04 ; P=0.002\right.$; Supplementary Table 24), or all lineages $\left(F_{1,33962}=23.13 ; P<0.0001 ;\right.$ Fig. 41 and Supplementary Table 27).

Regardless of how sympatry was measured, we found that phenotypic divergence is best predicted by evolutionary age, suggesting that most trait differences among lineages simply accumulate over evolutionary time as a result of processes such as genetic drift and ecological adaptation. This may explain why previous studies have reported the signature of ecological and reproductive character displacement to be absent ${ }^{24,25}$ or equivocal ${ }^{17}$ at the scale of clades and communities. However, it is striking that we find no evidence of divergent character displacement in bird beaks and songs, two traits that have played a central role in the development of character displacement theory ${ }^{1,2,15}$. Our phylogenetic comparative approach may have obscured individual cases of character displacement, yet our results indicate that this process is subtle or infrequent in ovenbirds, and fails to predict broad-scale patterns. We can rule out the possibility that our conclusions are specific to clades with weak species interactions as ovenbirds are generally territorial and predisposed to high levels of interspecific competition ${ }^{26}$. It is also unlikely that we have overlooked displacement because of statistical issues as our models have sufficient power to deal with noisy data, and even detect a signature of increased song similarity in sympatry.

This apparent convergence in songs is counter-intuitive from the perspective of classic character displacement theory, and opposite to the patterns generally assumed to be pervasive in birds ${ }^{2,27}$. Yet, it is consistent with the view that agonistic character displacement can drive adaptive convergence of signals mediating competitor recognition in multispecies systems ${ }^{9}$. This hypothesis is based on the idea that individuals with convergent agonistic signals have higher fitness because they are better at defending resources against both conspecific and heterospecific competitors. In birds, competition for food or territories can take place among relatively old sympatric lineages with partly overlapping ecological niches ${ }^{27}$. Although agonistic character displacement has rarely been demonstrated, our findings align with previous studies suggesting that social signals may converge owing to competition ${ }^{9,27,28}$, and that hybridization is then averted because receivers adapt to differentiate similar signals ${ }^{8}$. 

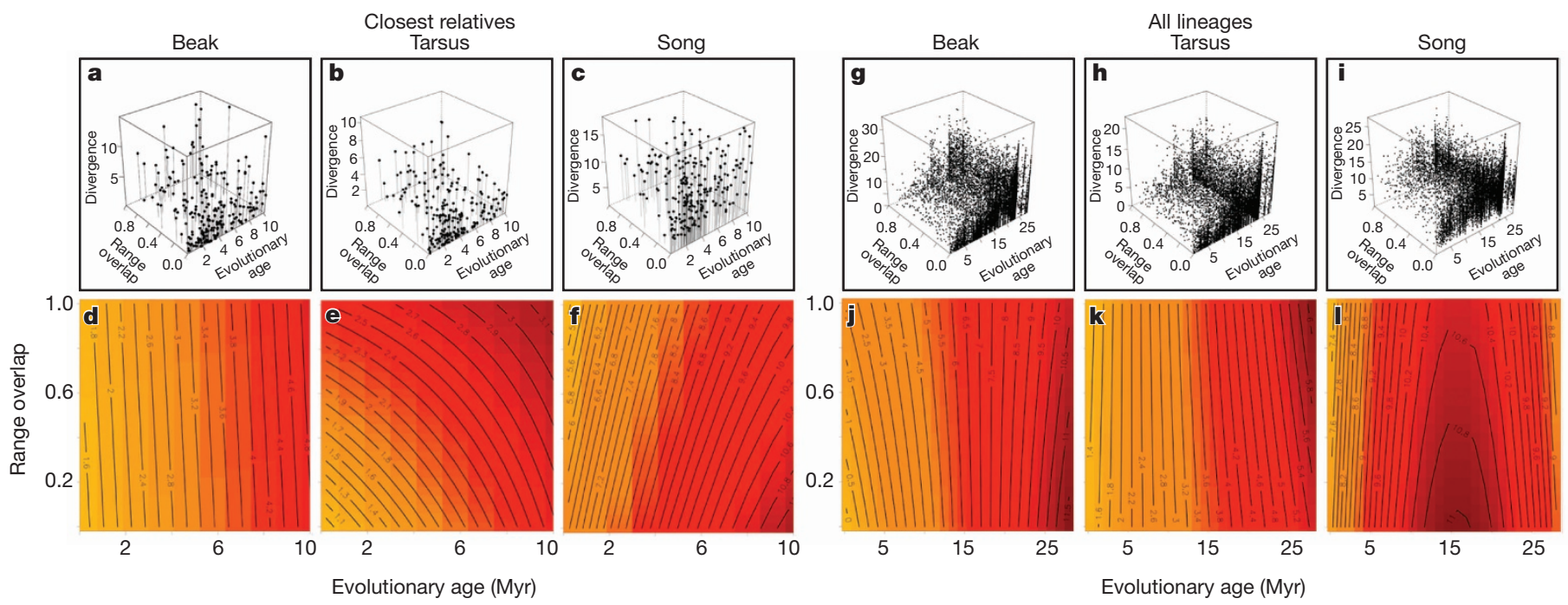

Figure $4 \mid$ The dynamics of phenotypic divergence across space and time. Divergence in ovenbird beaks, tarsi and songs between closest relatives $(\mathbf{a}-\mathbf{f})$ and all lineages $(\mathbf{g}-\mathbf{l})$ plotted as a function of proportional geographical range overlap and evolutionary age (Myr). Three-dimensional scatter plots of trait divergence $(\mathbf{a}-\mathbf{c} ; \mathbf{g}-\mathbf{i})$ show that data are noisy, as is expected with phenotypic trait evolution across large radiations. Because speciation tends to occur in allopatry, sampling of young, broadly sympatric lineages is relatively

An alternative explanation is that widespread hybridization occurs in ovenbirds, causing the introgression of genes coding for song structure. This seems unlikely given that hybridization should reduce molecular sequence divergence in sympatry, lowering the estimated age of interacting lineages (cf. Extended Data Fig. 4). Another possibility is that such lineages occur in more similar environments, potentially driving convergence through ecological adaptation. However, using coarse habitat categories, we found no significant difference in habitat divergence between sympatric and allopatric pairs of lineages (PLMM: $F_{5,258}=1.59, P=0.16$ ), largely because habitat niche divergence only occurs after an extended time-lag, regardless of geographical relationships (Extended Data Fig. 5). Indeed, previous studies have shown that even narrowly defined microhabitat niches are conserved over millions of years in ovenbirds, during which time sympatry is associated with niche divergence ${ }^{26}$. Given that a pattern of greater song similarity in sympatry is detected throughout this initial period of divergence (Fig. 4f), our results cannot simply be explained by acoustic adaptation to shared habitat. A related concern is that song divergence may be more bounded in sympatry than allopatry, generating spurious evidence of convergence. This may occur, for example, if sympatric ovenbirds tend to coexist in dense habitat where song divergence is potentially limited by constraints on signal transmission ${ }^{29}$. However, we found that song divergence was bounded regardless of geographical relationship, and although the constraint parameter $(\alpha)$ differed marginally in sympatry and allopatry, a strong signature of convergence was retained even after accounting for this discrepancy (Supplementary Table 10).

This signature may be produced by classic convergence (that is, decreasing trait differences over time), or else simply a failure to diverge. Although it is difficult to discriminate between these outcomes, both can be viewed as forms of convergence (Supplementary Information), and it seems likely that classic convergence contributes to the pattern. The hump-shaped trajectory of song divergence among closest relatives in sympatry (Fig. 3c), as indicated by a significant negative quadratic term in our models (Supplementary Tables 6 and 9), is not predicted by constraints on divergence. It is more consistent with accentuated convergence among older lineages owing to the accumulation of sympatry over time- that is, the average duration of coexistence increases gradually with age ${ }^{26}$. Furthermore, we assume that normal levels of divergence sparse (hence our preference for treating sympatry and allopatry as binary variables in models). Note that $\mathbf{g}-\mathbf{i}$ contain pairwise comparisons among all lineages and thus species contribute information to multiple data points. Heatmaps $(\mathbf{d}-\mathbf{f} ; \mathbf{j}-\mathbf{l})$ are shown to clarify the effect of proportional range overlap ( $y$ axis) and evolutionary time ( $x$ axis) on trait divergence (darker colours indicate greater divergence). For trait units, see Fig. 1c-e; for sample sizes, see Fig. 3.

occur during the allopatric phase after speciation in ovenbirds, and thus patterns of reduced divergence in sympatry are most probably produced by classic convergence after secondary contact. This possibility requires further testing with detailed field studies.

Although we do not measure species interactions directly, they offer the most likely explanation for song convergence in ovenbirds for three reasons. First, interactions among sympatric lineages are widespread, and often cause shifts in trait evolution ${ }^{5,12}$. Second, the contrast between non-converging beaks and tarsi (Fig. $4 \mathrm{~d}, \mathrm{e}$ ) and converging songs (Fig. 4f) suggests that opposing mechanisms regulate divergence in ecological versus social traits. Given that ecological trait divergence in ovenbirds is explained by adaptation to microhabitat or foraging substrate $e^{20}$, the implication is that song convergence may be driven by a separate, socially mediated mechanism. Third, the existence of remarkably non-divergent songs among interspecifically territorial ovenbird lineages several million years after speciation (Extended Data Fig. 9) is unlikely to arise through learning, hybridization, acoustic adaptation or bounded evolution, and yet is consistent with agonistic interactions $s^{9,27,28}$.

We have shown that uncorrected patterns of phenotypic variation are confounded by biases in the time available for trait differences to evolve, and exaggerate the role of character displacement at macroevolutionary scales. Once these biases are taken into account, we find no evidence that trait differences in coexisting ovenbirds are explained by species interactions, even among the 'most closely-allied forms' that Darwin $^{3}$ predicted would diverge as a result of 'the severest competition'. We propose that ecological and reproductive character displacement is restricted to cases of early secondary contact, particularly involving incipient species, and that this phenomenon is comparatively rare in the ancient, continental radiations that make up much of biodiversity.

Overall, our analyses support the hypothesis that lineages build up enough ecological and reproductive isolation during allopatry to bypass character displacement after secondary contact ${ }^{7,19,26}$. These results therefore challenge the assumption that ecological and reproductive character displacement are key microevolutionary mechanisms contributing to macroevolutionary patterns, including trait differences in communities and across adaptive radiations ${ }^{4,10,14}$. Moreover, we have shown that the same biases in evolutionary age that explain character differences in sympatric lineages potentially mask the role of convergent evolution. 
A pervasive pattern of phenotypic convergence is not new, even in radiations ${ }^{30}$, but our findings provide the first evidence that such outcomes can be driven by species interactions. We conclude that macroevolutionary patterns previously interpreted as character displacement should be re-analysed in an explicitly temporal framework, as doing so may show that mechanisms of character convergence are widespread.

\section{METHODS SUMMARY}

Focusing on 350 ovenbird lineages, we measured ecological traits (beak shape, tarsus length) from museum specimens, and a social trait (song structure) from digital recordings (see Fig. 1). Habitat preferences were scored from the literature. We used digital range polygons to quantify geographical range overlap as a continuous variable, and used a $20 \%$ range overlap threshold to convert this into a binary variable (sympatry/allopatry). To estimate the evolutionary time since divergence for all pairs of lineages, we produced a phylogenetic tree (Fig. 2) using standard techniques $^{6,20}$. We then used PLMMs to compare phenotypic divergence in sympatry versus allopatry, controlling for shared ancestry, evolutionary age and habitat differences. We focused at two taxonomic levels: closest relatives and the entire radiation. To assess whether our results were robust to different models of trait evolution, we also used phylogenetic generalized least squares (PGLS), and verified a new approach for accounting for best-fit models in both sympatry and allopatry using PLMMs. We examined the sensitivity of the results to several refinements, including restricting analyses to young lineages $(<6 \mathrm{Myr})$ or sister species, and treating proportional range overlap as a continuous variable. Data manipulation was conducted in $R$ version 3.0.1, PLMMs in ASReml-R, and PGLS analyses used $\mathrm{R}$ libraries 'ape' and 'nlme' (see Methods for all software references). For discussion and definition of the terms 'adaptive radiation', 'character displacement' and 'convergence' see Supplementary Information.

Online Content Any additional Methods, Extended Data display items and Source Data are available in the online version of the paper; references unique to these sections appear only in the online paper.

Received 7 March; accepted 8 November 2013.

Published online 22 December 2013.

1. Grant, P. R. \& Grant, B. R. Evolution of character displacement in Darwin's finches Science 313, 224-226 (2006).

2. Grant, B. R. \& Grant, P. R. Songs of Darwin's finches diverge when a new species enters the community. Proc. Natl Acad. Sci. USA 107, 20156-20163 (2010).

3. Darwin, C. R. On the Origin of Species (John Murray, 1859).

4. Simpson, G. G. Tempo and Mode in Evolution (Columbia Univ. Press, 1944)

5. Pfennig, D. W. \& Pfennig, K. S. Character displacement and the origins of diversity. Am. Nat. 176, S26-S44 (2010).

6. Weir, J. T. \& Price, T. D. Limits to speciation inferred from times to secondary sympatry and ages of hybridizing species along a latitudinal gradient. Am. Nat 177, 462-469 (2011)

7. Price, T. D. The roles of time and ecology in the continental radiation of the Old World leaf warblers (Phylloscopus and Seicercus). Phil. Trans. R. Soc. B 365, 1749-1762 (2010).

8. Seddon, N. \& Tobias, J. A. Character displacement from the receiver's perspective: species and mate recognition despite convergent signals in suboscine birds. Proc R. Soc. Lond. B 277, 2475-2483 (2010).

9. Grether, G. F., Losin, N., Anderson, C. N. \& Okamoto, K. The role of interspecific interference competition in character displacement and the evolution of competitor recognition. Biol. Rev. Camb. Philos. Soc. 84, 617-635 (2009).

10. Reznick, D. N. \& Ricklefs, R. E. Darwin's bridge between microevolution and macroevolution. Nature 457, 837-842 (2009).

11. Brown, W. L. \& Wilson, E. O. Character displacement. Syst. Zool. 5, 49-64 (1956).
12. Pfennig, K. S. \& Pfennig, D. W. Character displacement: ecological and reproductive responses to a common evolutionary problem. Q. Rev. Biol. 84 253-276 (2009).

13. Gavrilets, S. \& Losos, J. B. Adaptive radiation: contrasting theory with data. Science 323, 732-737 (2009).

14. Dayan, T. \& Simberloff, D. Ecological and community-wide character displacement: the next generation. Ecol. Lett. 8, 875-894 (2005).

15. Schoener, T.W. The evolution of bill size differences among sympatric congeneric species of birds. Evolution 19, 189-213 (1965).

16. Sætre, G. P. et al. A sexually selected character displacement in flycatchers reinforces premating isolation. Nature 387, 589-592 (1997).

17. Davies, T., Meiri, S., Barraclough, T. \& Gittleman, J. Species co-existence and character divergence across carnivores. Ecol. Lett. 10, 146-152 (2007).

18. Connell, J. H. Diversity and the coevolution of competitors, or the ghost of competition past. Oikos 35, 131-138 (1980).

19. Rundell, R. J. \& Price, T. D. Adaptive radiation, non-adaptive radiation, ecological speciation and non-ecological speciation. Trends Ecol. Evol. 24, 394-399 (2009).

20. Derryberry, E. P. et al. Lineage diversification and morphological evolution in a large-scale continental radiation: the Neotropical ovenbirds and woodcreepers (Aves: Furnariidae). Evolution 65, 2973-2986 (2011).

21. Seddon, N. Ecological adaptation and species recognition drive vocal evolution in Neotropical suboscine birds. Evolution 59, 200-215 (2005).

22. Tobias, J. A. et al. Song divergence by sensory drive in Amazonian birds. Evolution 64, 2820-2839 (2010)

23. Derryberry, E. P. et al. Correlated evolution of beak morphology and song in the Neotropical woodcreeper radiation. Evolution 66, 2784-2797 (2012).

24. Huntley, J. W. et al. Testing limiting similarity in Quaternary terrestrial gastropods. Paleobiology 34, 378-388 (2008).

25. Monroe, M. J. Does competition drive character differences between species on a macroevolutionary scale? J. Evol. Biol. 25, 2341-2347 (2012)

26. Pigot, A. L. \& Tobias, J. A. Species interactions constrain geographic range expansion over evolutionary time. Ecol. Lett. 16, 330-338 (2012).

27. Tobias, J. A. \& Seddon, N. Signal design and perception in Hypocnemis antbirds: evidence for convergent evolution via social selection. Evolution 63, 3168-3189 (2009).

28. Laiolo, P. Interspecific interactions drive cultural co-evolution and acoustic convergence in syntopic species. J. Anim. Ecol. 81, 594-604 (2012).

29. Weir, J. T., Wheatcroft, D. \& Price, T. The role of ecological constraint in driving the evolution of avian song frequency across a latitudinal gradient. Evolution 66, 2773-2783 (2012).

30. Muschick, M., Indermaur, A. \& Salzburger, W. Convergent evolution within an adaptive radiation of cichlid fishes. Curr. Biol. 22, 2362-2368 (2012).

Supplementary Information is available in the online version of the paper.

Acknowledgements We thank G. Grether, J. Hadfield, S. Nakagawa, A. Phillimore A. Pigot, R. Ricklefs, G. Thomas and S. West for comments and discussion. We are also indebted to the many individuals who collected specimens, tissue samples and sound recordings, and to numerous institutions (particularly the Macaulay Library, Cornell University) for granting access to this material. Complete acknowledgements and data sets are provided in the Supplementary Information. This research was supported by the John Fell Fund (to J.A.T.), the Browne Fellowship, Queen's College, Oxford, and Vetenskapsrådet (to C.K.C.), the National Science Foundation (to R.T.B.) and the Royal Society (to N.S.).

Author Contributions J.A.T. and N.S. conceived and designed the study, compiled and analysed song data, and integrated all data sets; S.C. provided morphometric data; E.P.D., S.C. and R.T.B. conducted molecular sequencing and phylogenetic analyses; C.C. designed and conducted statistical analyses, with significant input from N.S.; N.S J.A.T. and C.C. produced figures and tables; J.A.T. prepared and edited the manuscript with input from all authors.

Author Information Nuclear and mitochondrial DNA sequences for all lineages have been deposited in GenBank under accession numbers given in Supplementary Data 1. Reprints and permissions information is available at www.nature.com/reprints. The authors declare no competing financial interests. Readers are welcome to comment on the online version of the paper. Correspondence and requests for materials should be addressed to J.A.T. (joseph.tobias@zoo.ox.ac.uk). 


\section{METHODS}

Phylogenetic framework. We compiled published molecular sequence data (three mitochondrial genes and one nuclear intron) for 279 (95\%) of 295 extant ovenbird species, along with six intraspecific lineages ${ }^{20}$. To capture the complete time span of phenotypic divergence in this radiation, we sequenced the same genes for a further 65 intraspecific lineages representing the first stages of trait divergence. We constructed a maximum clade credibility (MCC) tree (Fig. 2) for all 350 lineages (Supplementary Data 1), and used a relaxed molecular clock to calibrate branch lengths. This allowed us to calculate absolute times since divergence for all pairs of lineages (using relative ages or estimates of molecular divergence would produce identical results).

Data collection. To quantify ecological traits, we measured four morphometric characters (beak length, depth and width; tarsus length; Fig. 1) from museum specimens $(\sim 5,000$ measures; mean \pm s.d. $=3.7 \pm 1.5$ specimens per lineage) . To quantify social traits, we used the MatLab signal processing toolbox (Mathworks) to generate broadband spectrograms from 1,854 digital recordings of ovenbird songs, and then extracted a total of 32 acoustic variables (Supplementary Table 1) using a custom script ( $\sim 50,000$ acoustic measurements; $5.3 \pm 3.0$ individuals sampled per lineage). To ensure that phenotypic comparisons were made between relevant populations, we sampled from regions of overlap in sympatric closest relatives (see below). For a full list of sources for museum specimens and acoustic data, see Supplementary Data 1.

Tarsus divergence was calculated as the absolute value of the difference between measurements. For the multivariate beak and song data sets, we conducted a phylogenetic principal components analysis on the correlation matrices of lineage mean values (log-transformed). Beak measurements were reduced to a single component representing beak morphology $\left(\mathrm{PCl}_{\text {beak }}\right)$, which explained $75.8 \%$ of the variation and with which all variables had high correlation coefficients $(>0.8)$. For acoustic traits, principal components analysis extracted 14 uncorrelated principal components (factor loadings in Supplementary Data 1). We quantified divergence between lineages as the Euclidean distance between the species centroids using the 'dist' function in $R$.

Habitat niche. We used standard published sources to classify primary habitat of all lineages as (1) closed-canopy forest, (2) open-canopy woodland and shrublands or (3) grasslands and desert (Fig. 2). We used this scoring system to provide an index of ecological divergence for each pairwise comparison between lineages. We included all ecological contrasts in models: 1 versus $1 ; 2$ versus $2 ; 3$ versus 3 ; 1 versus $2 ; 2$ versus $3 ; 1$ versus 3 . Because ecological niches have a strong phylogenetic signal, moderate and large contrasts were mainly associated with older nodes in the ovenbird phylogeny (Extended Data Fig. 5).

Geographical relationships. We used digital range polygons downloaded from NatureServe ${ }^{31}$ to quantify overlap (sympatry) for all pairwise comparisons between lineages. Where our data set contained more than one intraspecific lineage, we subdivided range polygons according to published range descriptions for each lineage. Note that all intraspecific lineages were distributed allopatrically, with clear biogeographical limits (for example, separated by range disjunctions). We used ArcGIS 9.1 (ESRI) to calculate degree of overlap as the proportion of the smaller range that occurred in the larger range.

We treated geographical range overlap in two ways. First, we converted it to a binary variable by categorizing each pairwise comparison as either allopatric or sympatric, following established thresholds ${ }^{32,33}$ : allopatric species were defined as those with mutually exclusive or narrowly $(<20 \%)$ overlapping breeding ranges; sympatric species were defined as those with broadly $(>20 \%)$ overlapping breeding ranges. We checked results against published range descriptions and specimen locality data, and revised geographical relationships when it was clear that range polygons were misleading. Second, to explore the relationship between phenotypic divergence and extent of sympatry in each pair of species, we treated range overlap as a continuous variable (proportion of smaller range overlapped by larger range). Statistical analyses. General approach. To model the dynamics of character divergence, we used PLMMs with restricted maximum likelihood estimation. We tested whether there were overall differences in trait divergence between allopatric and sympatric lineages, while controlling for time since lineage divergence, by fitting sympatry (two-level factor: allopatry, sympatry), habitat differences (6-level factor: all comparisons between three habitat types), evolutionary age and the square of evolutionary age (covariate) as fixed effects. The square of evolutionary age, a quadratic term, was fitted to account for curvilinear patterns of trait divergence over time, including bounded evolution (see Extended Data Fig. 10 for validation). We also fitted interactions between sympatry*evolutionary age and sympatry*the square of evolutionary age, thereby estimating separate slopes for allopatric and sympatric comparisons and allowing us to test whether allopatric and sympatric lineages differed in linear and curvilinear trait divergence over time. All covariates were mean centred and standardized to unit variance ( $z$-transformed) before analyses. Response variables were transformed to ensure model residuals were normally distributed and homoscedastic.

As some lineages were represented several times in each data set (Supplementary Table 2), the non-independence of data points was taken into account by fitting both the focal lineage (labelled as lineage 1 in analysis tables) and the lineage they were compared with (lineage 2 ) as random effects. To account for the influence of shared ancestry in our models, we included the phylogenetic covariance matrix of the ancestral nodes between lineages as a random effect. The significance of fixed effects was examined using Wald-type $F$-tests with denominator degrees of freedom calculated following ref. 34 . We first tested the significance of interactions using full models with all terms included, and then removed higher-order interactions to test the main effects in each model. We assessed the significance of random effects using log-likelihood ratio tests, with all fixed effects and their interactions included in models ${ }^{35}$. All data manipulation was conducted in $R$ version 3.0.1 (http://cran.r-project.org), and PLMMs were performed in ASReml- ${ }^{36}$. Further details on accounting for non-independence in data sets and error in calculating evolutionary age are given in Supplementary Information.

Specific analyses (described in full under the same numbering system in Supplementary Information) were as follows.

Analysis 1. To test for character displacement in closest relatives independent of evolutionary age, we analysed Euclidean distance in tarsus length, beak morphology and song structure using separate linear mixed models (LMMs) conducted on all pairs of closest relatives in sympatry $(n=270$ pairs $)$ and allopatry $(n=249$ pairs), with sympatry as a fixed factor (Supplementary Table 3 ).

Analysis 2.1. To test for character displacement in closest relatives controlling for habitat and time, we ran the models described in analysis 1 including habitat differences and evolutionary age as covariates. Interactions between sympatry and all other fixed effects were included (Supplementary Tables 4-6). We calculated the error in estimating evolutionary age as the variance in age for each pair of lineages obtained from the 1,000 posterior trees that were used to calculate the MCC tree, and included this error as a random effect in PLMMs.

Analysis 2.2. PLMMs assume a Brownian motion model of evolution, which may not be valid if divergence is constrained, or rates of divergence are variable over evolutionary time. We therefore examined which model of trait evolution best explained variation in tarsi, beaks and songs, and then accounted for the best-fit model using PGLS and multi-response PLMMs fitted with phylogenetic covariance matrices transformed according to different models of evolution ${ }^{37}$. Specifically, we tested Brownian motion models of evolution against (1) Ornstein-Uhlenbeck and (2) accelerated/decelerated models of evolution. We examined these different models of evolution in the following way.

First, we compared the results of three different methods for assessing evolutionary models: PGLS (R packages 'ape' and 'nlme ${ }^{38,39}$ ), maximum likelihood estimation ('fitContinuous' in R package 'Geiger' ${ }^{40}$ ) and PLMMs fitted with the MCC tree transformed under different models of evolution in 'Geiger'. We used data on raw trait values so that equivalent models could be implemented with all techniques, and selected best-fit models for each method using Akaike information criterion values (Supplementary Table 7). Second, given that (1) all methods gave similar results and (2) ASReml-R is the only technique that allows repeated measurement of lineages, we examined best-fit models of evolution in main analyses (closest relatives and all lineages) using PLMMs with the same fixed and random effects as analysis 2.1

We found that divergence in ecological traits followed a Brownian motion model of evolution; thus for these traits the assumption of Brownian motion evolution in PLMMs was valid. However, the best-fit model for song evolution was OrnsteinUhlenbeck, suggesting that bounded evolution might have influenced our results We therefore accounted for the evolutionary model that best explained song divergence using PLMMs. This had no effect on the results of analyses on closest relatives (Supplementary Table 9) or all lineages (Supplementary Table 27). To examine whether song convergence was explained by increased constraints on song divergence in sympatry, we used multiple-response PLMMs with two phylogenetic covariance matrices fitted, one for sympatric lineages and one for allopatric lineages. This allowed us to fit different models of evolution for allopatric and sympatric comparisons. We linked allopatric comparisons to a phylogenetic covariance matrix formed from the MCC tree after it had been transformed according to the best-fit model of evolution estimated using only data on allopatric lineages. Sympatric comparisons were linked to a different phylogenetic covariance matrix that had been constructed from the MCC tree transformed according to the best-fit model of evolution estimated only from data on sympatric lineages. Fixed and random effects were entered as in analysis 2.1. Incorporating the model of evolution that best explained divergence in song structure in allopatry and sympatry did not alter the conclusions of our main analyses (Supplementary Table 10).

Analysis 2.3. To investigate the sensitivity of our results to taxonomy, we re-ran analysis 2.1 excluding intraspecific lineages (Supplementary Tables 11-13). 
Analysis 2.4. To test for character displacement in the earliest stages of divergence among closest relatives, we re-ran analysis 2.1 including only pairs of sister lineages and accounting for best-fit models of evolution (Supplementary Tables 14 and 15).

Analysis 2.5. To test for character displacement in the period of divergence showing apparent character displacement in Fig. 3, we re-ran analysis 2.1 including only those lineages $<6 \mathrm{Myr}$ (Supplementary Tables 16-18).

Analysis 3 . To test for character displacement among all 350 ovenbird lineages, we ran models described in analysis 2.1 including pairwise comparisons between all lineages, excluding those between subfamilies, and controlling for best-fit model of trait evolution (Supplementary Tables 19-21).

Analysis 4 . To investigate the sensitivity of our results to using range overlap as a binary variable (sympatry/allopatry), we re-ran models with the proportion of range overlap as a continuous variable (fixed effect; covariate), and controlling for best-fit model of trait evolution (Supplementary Tables 22-27 and Fig. 4).

Analysis 5. We used four main approaches to assess the validity of PLMMs as a method for testing character displacement. First, we used permutation tests repeated 1,000 times, each time randomly shuffling the response variable to assess whether parameter estimates were biased by the structure of our data (Extended Data Fig. 6). Second, we reconstructed our trait data sets by simulating divergence under the model of evolution that best explained trait divergence in analysis 2.2 (Supplementary Table 8). This provided an extra test of whether our parameter estimates were confounded by data structure, and showed our results were robust to differences in the model of evolution (Extended Data Fig. 7). Third, we simulated a range of differences between allopatric and sympatric lineages (each run 5,000 times) and then, fourth, showed that PLMMs recovered these simulated differences under both Brownian motion and Ornstein-Uhlenbeck models (Extended Data Fig. 8). All trait simulations were performed using rTraitCont ( $\mathrm{R}$ package 'ape').

31. Ridgely, R. S. et al. Digital Distribution Maps of the Birds of the Western Hemisphere v. 2.1 (NatureServe, 2005)

32. Chesser, R. T. \& Zink, R. M. Mode of speciation in birds: a test of Lynch's method. Evolution 48, 490-497 (1995).

33. Lynch, J. D. in Speciation and its Consequences (eds Otte, D. \& Endler, J. A.) 527-553 (Sinauer, 1989).

34. Kenward, M. G. \& Roger, J. H. Small sample inference for fixed effects from restricted maximum likelihood. Biometrics 53, 983-997 (1997)

35. Self, S. G. \& Liang, K.-Y. Asymptotic properties of maximum likelihood estimators and likelihood ratio tests under nonstandard conditions. J. Am. Stat. Assoc. $\mathbf{8 2}$ 605-610 (1987)

36. Gilmour, A. R., Gogel, B. J., Cullis, B. R., Welham, S. J. \& Thompson, R. ASRemI User Guide, Release 3.0 (VSN International, 2009).

37. Hadfield, J. D. \& Nakagawa, S. General quantitative genetic methods for comparative biology: phylogenies, taxonomies, meta-analysis and multi-trait models for continuous and categorical characters. J. Evol. Biol. 23, 494-508(2010).

38. Paradis, E., Claude, J. \& Strimmer, K. APE: analyses of phylogenetics and evolution in R language. Bioinformatics 20, 289-290 (2004).

39. Pinheiro, J., Bates, D., DebRoy, S. \& Sarkar, D. Package nlme: Linear and Nonlinear Mixed Effects Models, v. 3.1-109 (R-core, 2013)

40. Harmon, L. J., Weir, J. T., Brock, C. D., Glor, R. E. \& Challenger, W. GEIGER: investigating evolutionary radiations. Bioinformatics 24, 129-131 (2008). 


\section{RESEARCH LETTER}

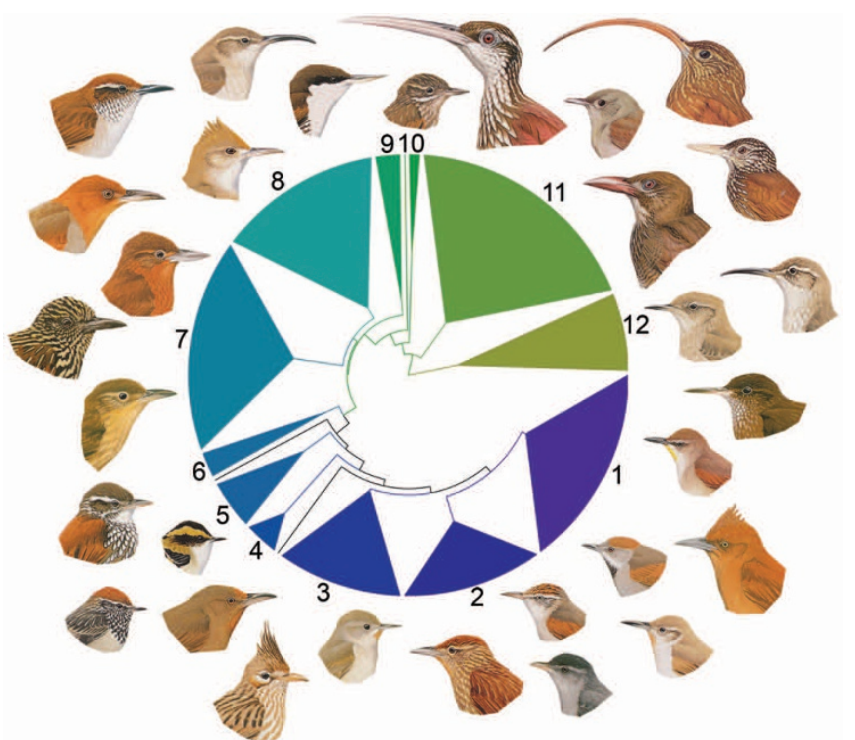

Extended Data Figure $1 \mid$ Phenotypic radiation. Variation in beak morphology and plumage across 350 lineages in 12 major clades of ovenbirds (species richness in each clade is represented by distance on the circumference of the phylogram; clades are coloured to facilitate interpretation). Numbered clades (species names running clockwise) are as follows: 1, Synallaxini, clade A (Certhiaxis cinnamomeus, Pseudoseisura cristata, Synallaxis azarae, Pseudasthenes cactorum); 2, Synallaxini, clade B (Cranioleuca hellmayri, Acrobatornis fonsecai, Thripophaga macroura); 3, Synallaxini, clade C (Asthenes pyrrholeuca, Coryphistera alaudina); 4, Phacellodomus (Phacellodomus erythrophthalmus); 5, Leptasthenura (Leptasthenura xenothorax, Aphrastura spinicauda); 6, Margarornis/Premnoplex (Margarornis squamiger); 7, Philydorini (Automolus ochrolaemus, Thripadectes flammulatus, Syndactyla ucayalae, Hylocryptus erythrocephalis, Clibanornis dendrocolaptoides); 8, Furnariini (Furnarius cristatus, Upucerthia jelskii); 9, Ochetorhynchus (Pygarrhichas albogularis); 10, Xenops (Xenops tenuirostris); 11, Dendrocolaptinae (Nasica longirostris, Sittasomus griseicapillus, Campylorhamphus trochilirostris, Hylexetastes stresemanni, Dendroplex picus); 12, Sclerurinae (Geositta tenuirostris, Geositta antarctica, Sclerurus guatemalensis). Illustrations reproduced with the permission of Lynx Edicions. 


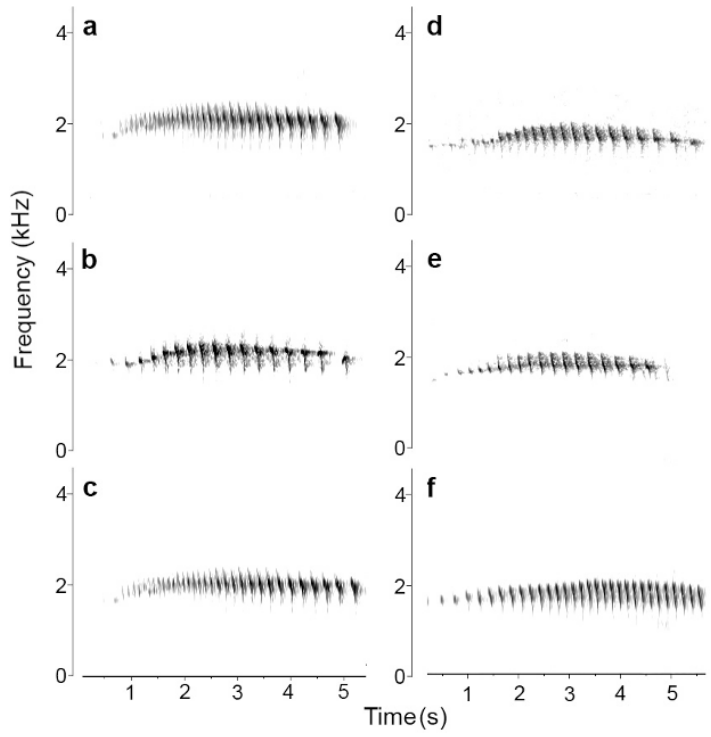

Extended Data Figure $2 \mid$ Stereotypy in ovenbird songs. Examples of single songs given by six different individuals of one species of ovenbird (Xiphorhynchus guttatus) to illustrate typical levels of stereotypy of acoustic structure in a song sample in our data set, as well as the relative simplicity of suboscine songs (compared with the songs of oscine songbirds). Songs recorded in Bolivia (a-c) and Peru (d-f) were used to generate spectrograms (time versus frequency) using Raven Pro (settings: window $=$ Hann, bandwidth $=256 \mathrm{~Hz}$, fast Fourier transform $=1,024$, overlap $=0.875)$. Catalogue numbers of recordings: (a) XC2297, (b) XC64610, (c) XC1756, (d) JATXiphGutt02, (e) JATXiphGutt08, (f) XC84 (see Supplementary Data 1). 


\section{RESEARCH LETTER}
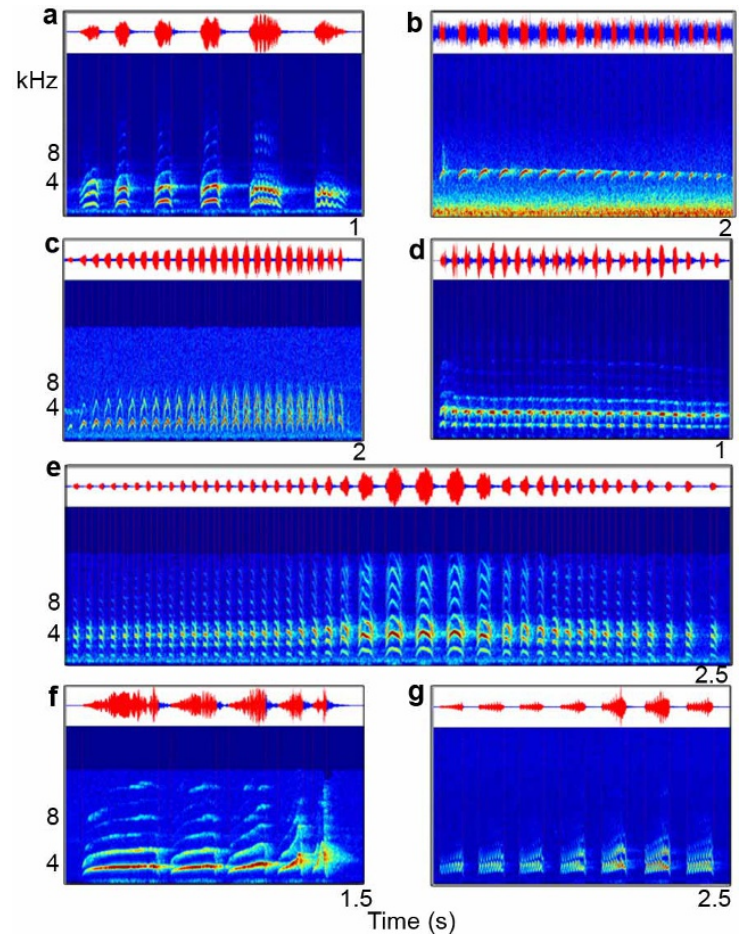

Extended Data Figure $3 \mid$ Representative songs from the seven major clades of ovenbirds. a, Synallaxini, clade A: Synallaxis erythrothorax (catalogue number ML7048, country El Salvador); b, Synallaxini, clade B: Cranioleuca curtata (ML120989, Bolivia); c, Synallaxini, clade C: Asthenes modesta (JAT99129, Bolivia); d, Philydorini: Automolus rufipileatus consobrina (ML66235, Venezuela); e, Furnariini: Cinclodes aricomae (JAT99138, Bolivia); f, Dendrocolaptinae: Campylorhamphus trochilirostris hellmayri (XC48901, Argentina); g, Sclerurinae: Geositta rufipennis rufipennis (ML46401, Argentina). Shown are waveforms (time versus amplitude, above) and spectrograms (time versus frequency) generated in MATLAB. Vertical dashed red lines (visible at high magnification) show note onset and offset. 

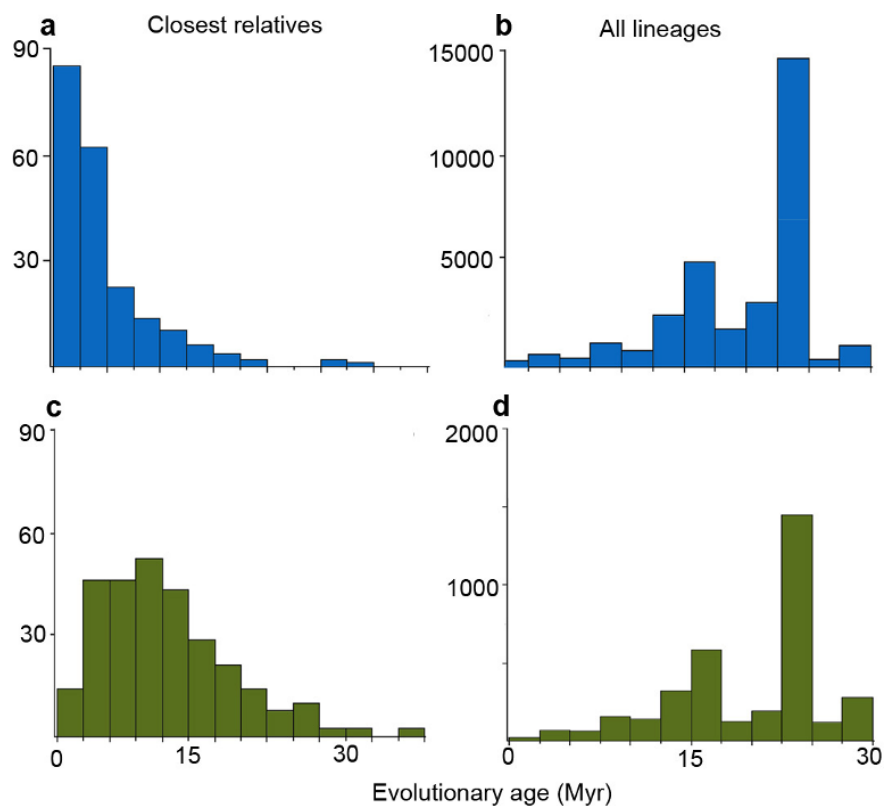

Extended Data Figure $4 \mid$ Evolutionary ages of ovenbird lineages.

Histograms show the frequency distribution of ages in allopatry

(blue: a, b) versus sympatry (green: $\mathbf{c}, \mathbf{d}$ ) for pairs of closest relatives (a, c)

and for all pairs of lineages $(\mathbf{b}, \mathbf{d})$. The reduced age of allopatric relatives is most evident in a: only seven (2.6\%) of the closest sympatric lineages were estimated to be $<2 \mathrm{Myr}$ old as opposed to 79 (31.6\%) of closest allopatric lineages.

Data are from all 350 lineages, excluding pairwise comparisons across subfamilies (that is, restricting to comparisons in Furnariinae,

Dendrocolaptinae and Sclerurinae). 


\section{RESEARCH LETTER}
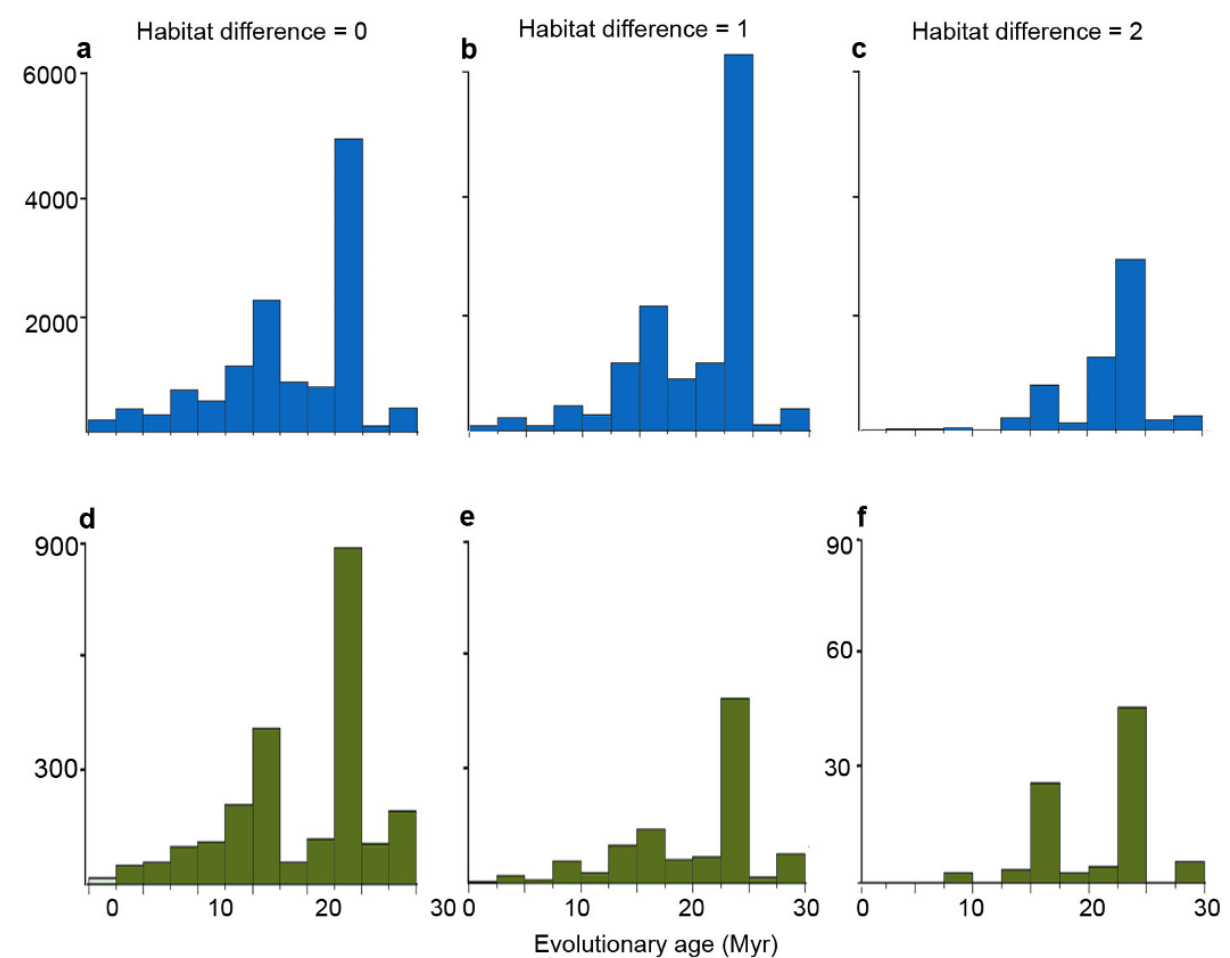

Extended Data Figure 5 Relationship between habitat divergence and evolutionary age in ovenbirds. Histograms show patterns in allopatry (blue: $\mathbf{a}-\mathbf{c}$ ) versus sympatry (green: $\mathbf{d}-\mathbf{f}$ ), plotted against evolutionary age: a, d, lineages occupying the same habitat (that is, both in closed, semi-open or open habitats); b, e, lineages occupying moderately divergent habitats (closed versus semi-open, or semi-open versus open); c, f, lineages occupying very different habitats (closed versus open). Data are from all 350 lineages, excluding pairwise comparisons across subfamilies (that is, restricting to comparisons in Furnariinae, Dendrocolaptinae and Sclerurinae). 

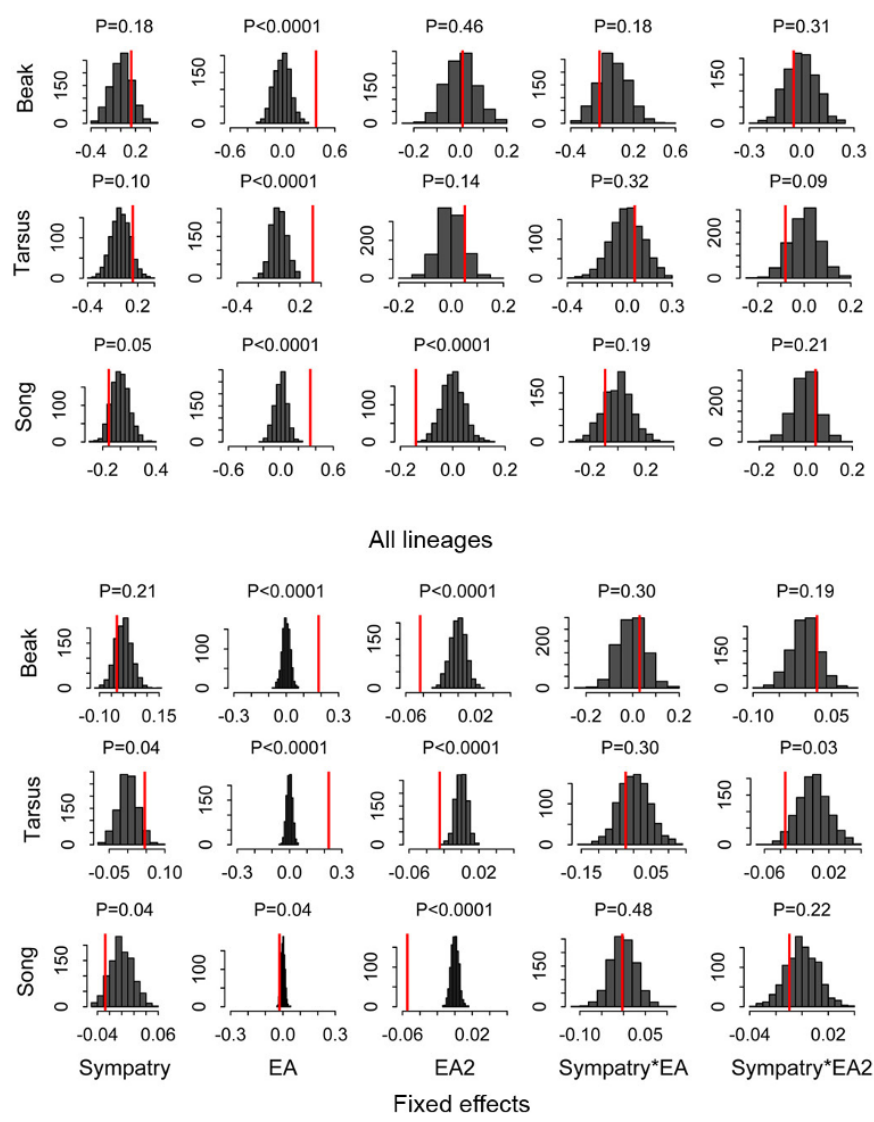

Extended Data Figure $6 \mid$ Permutation tests examining the influence of data structure and response variable distribution on fixed effects. Histograms show the frequency distributions of parameter estimates ( $x$ axis) obtained by randomly shuffling the response variable and re-running models 1,000 times. This procedure was applied to each fixed effect included in PLMMs, first on all pairs of closest relatives (upper panels) and then on all lineages (lower panels). Red lines show the parameter estimates obtained in our main analyses on observed data; $P$ values refer to the proportion of permutations for which the parameter estimate obtained from observed data was greater (or less) than the randomly generated response. In all analyses, permutation $P$ values were very similar to those obtained using $F$-tests (close relatives: Supplementary Tables 4-6; all lineages: Supplementary Tables 18-20), confirming that our results were not explained by biases in the structure of our data sets and variation in the distribution of our response variables. 


\section{RESEARCH LETTER}
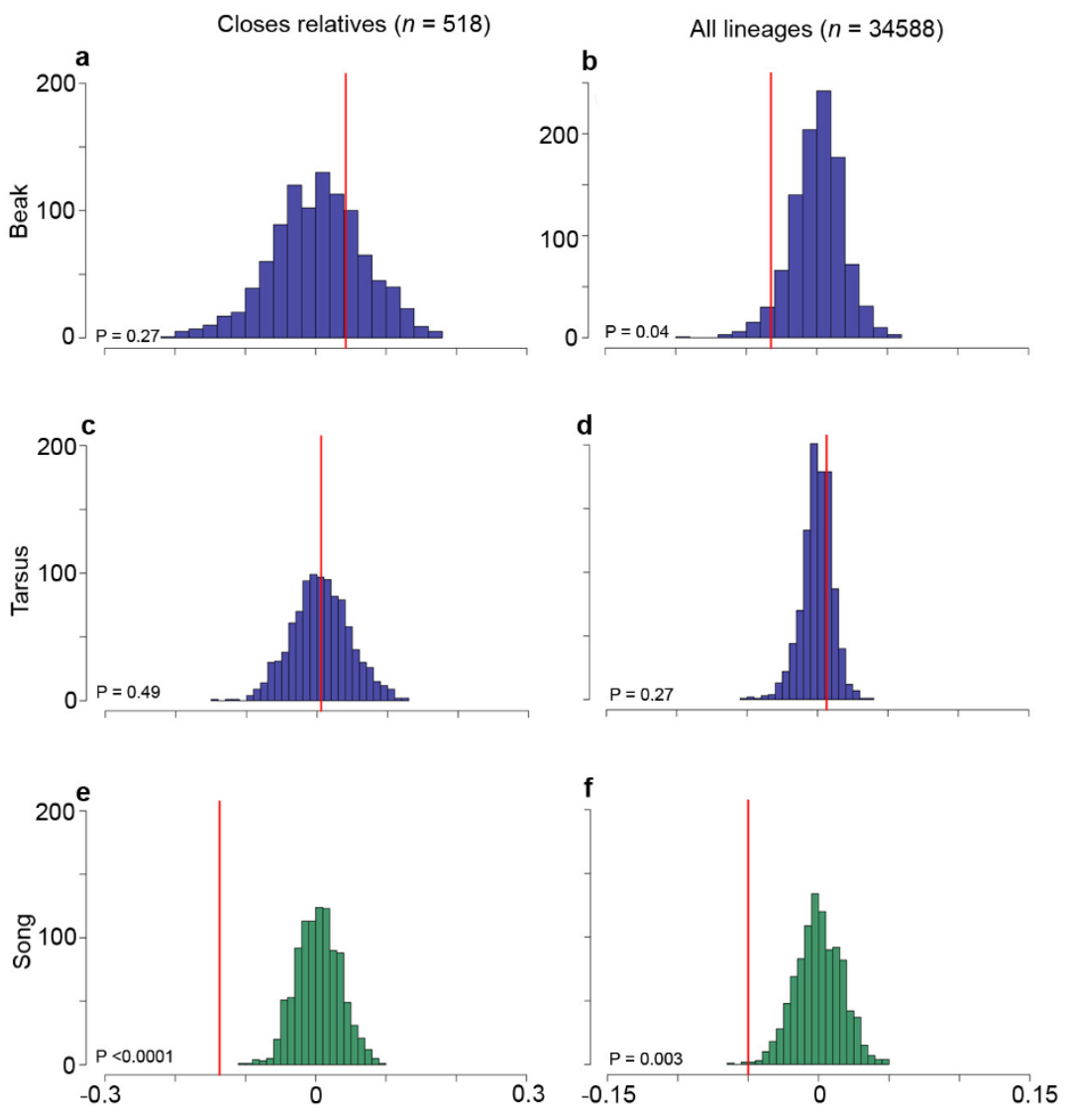

Estimated difference between allopatric and sympatric lineages

Extended Data Figure $7 \mid$ Comparing observed and simulated data sets under best-fit models of evolution. Histograms show estimated differences between allopatric and sympatric lineages of ovenbird in beak morphology $(\mathbf{a}, \mathbf{b})$, tarsus length $(\mathbf{c}, \mathbf{d})$ and song structure $(\mathbf{e}, \mathbf{f})$, comparing values obtained in our main analyses (red line; Supplementary Tables 4-6 and 18-20) with data simulated under best-fit models of evolution. Different colours denote simulated trait data following a Brownian motion model of evolution (blue), and an Ornstein-Uhlenbeck model (green). $P$ values are the proportion of simulations $(n=1,000)$ where the estimated difference obtained from our main analysis was greater (or less) than the estimate from the simulated data. For all traits and data sets, we found that $P$ values from simulations corresponded to those in main analyses. Note that convergence in beaks is marginally significant and restricted to the all-lineages analysis $(\mathbf{b})$, whereas convergence in songs is strongly significant and consistent across both analyses $(\mathbf{e}, \mathbf{f})$. 


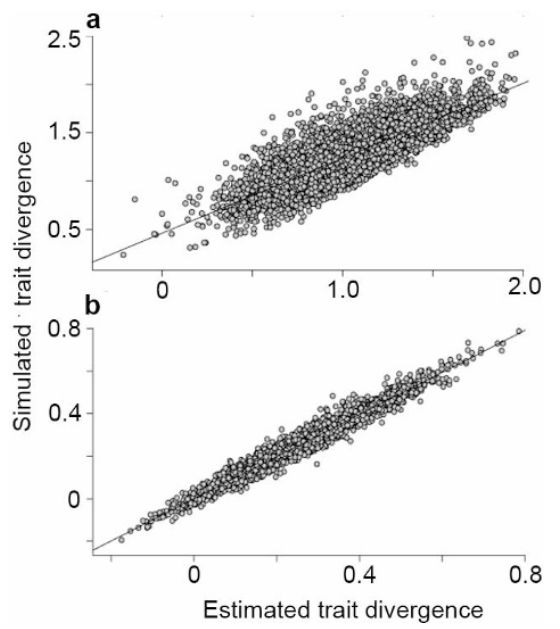

Extended Data Figure $8 \mid$ Recovery of simulated trait divergence under different models of trait evolution. Plots compare simulated and observed trait divergence between pairs of closely related ovenbird lineages (allopatric and sympatric lineages pooled) under two models of evolution using PLMMs: a, Brownian motion; b, Ornstein-Uhlenbeck. These 1:1 relationships demonstrate that PLMMs were able to recover differences simulated under both evolutionary models detected in our data sets. Scatter is reduced under Ornstein-Uhlenbeck because divergence is constrained. 


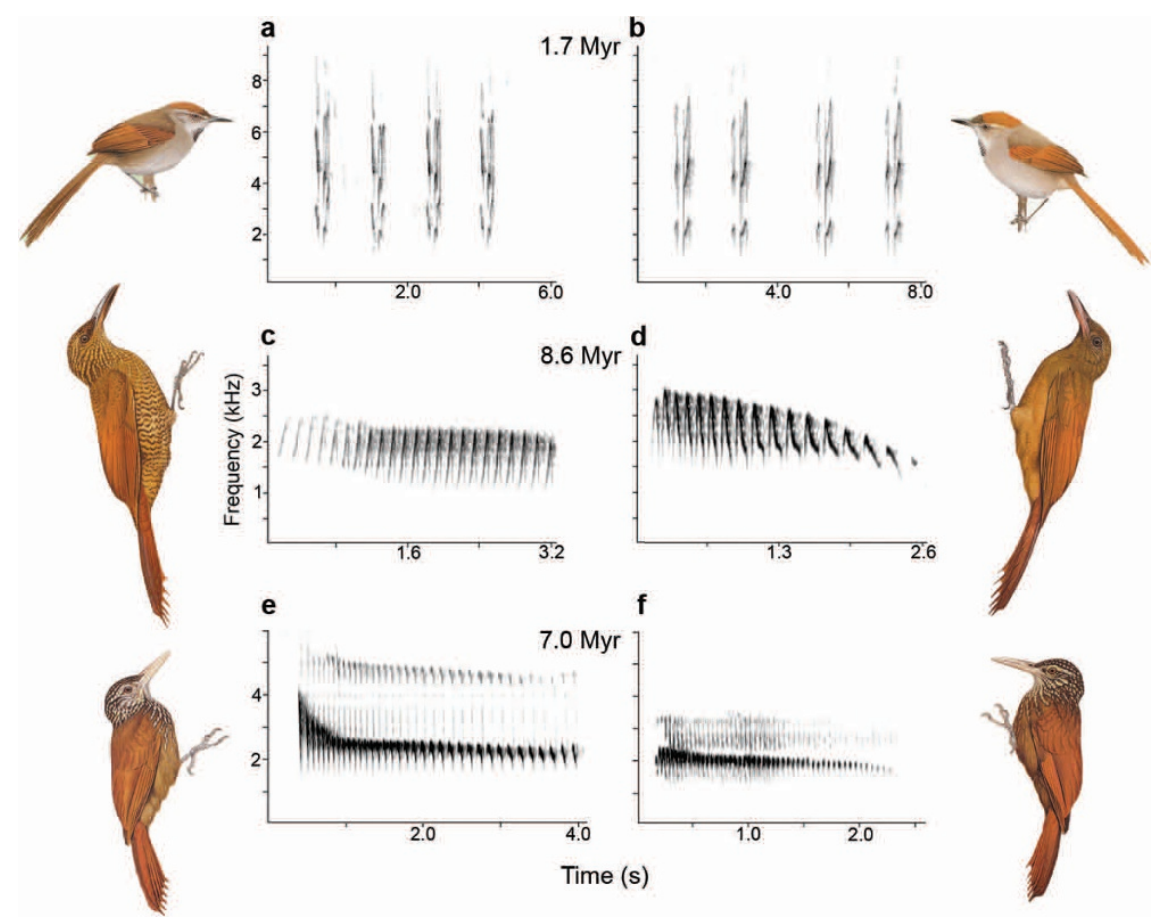

Extended Data Figure 9 | Examples of ovenbird lineages with similar songs in sympatry. a, Synallaxis frontalis and $\mathbf{b}$, Synallaxis azarae superciliosus; c, Dendrocolaptes picumnus picumnus and d, Dendrocolaptes certhia concolor; e, Dendroplex picus and $\mathbf{f}$, Dendroplex kieneri. For each pair of recordings (taken at the same locality), spectrograms show that songs of each lineage are species specific, but highly similar in key acoustic traits (for example, peak frequency or pace) to their closest sympatric relative. Figures are annotated with the approximate time in millions of years since divergence. Illustrations reproduced with the permission of Lynx Edicions. 

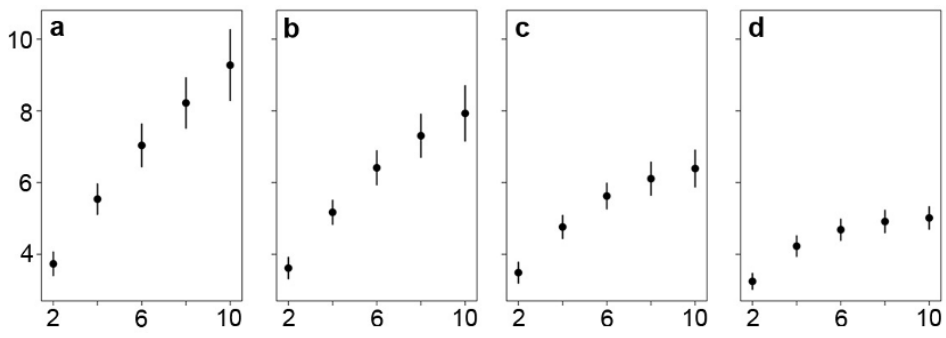

Extended Data Figure 10 Interpreting linear and quadratic terms of evolutionary age. Trait data were simulated over 100 randomly generated trees specifying four different models of evolution: a, Brownian motion; b, Ornstein-Uhlenbeck $(\alpha=0.1)$; c, Ornstein-Uhlenbeck $(\alpha=0.3)$; d, Ornstein-Uhlenbeck $(\alpha=0.6)$. Pairwise genetic ( $x$ axis: evolutionary age) and trait differences ( $y$ axis: trait divergence) between taxa were extracted from

simulated data. Trait data and trees were simulated using the $\mathrm{R}$ package 'ape' with the functions rTraitCont and rtree. Plots show that the Brownian motion model predicts a linear term and the Ornstein-Uhlenbeck model predicts a quadratic term, particularly when the constraint parameter increases in strength. 